\title{
Negotiation in Automated E-Coaching
}

\section{an Application in Mobile Insomnia Treatment}

\author{
Robbert Jan Beun \\ Utrecht University \\ Princetonplein 5, Utrecht \\ The Netherlands \\ r.j.beun@uu.nl
}

\author{
Fiemke Griffioen-Both \\ Utrecht University \\ Princetonplein 5, Utrecht \\ The Netherlands \\ fiemke@gmail.com
}

\author{
Jaap Lancee \\ University of Amsterdam \\ PO box 15933, Amsterdam \\ The Netherlands \\ j.lancee@uva.nl
}

\begin{abstract}
In this paper, we present the design of a negotiation protocol for behaviour change exercises that are supported by automated e-coaching systems. Negotiation is introduced as an interactive persuasive strategy with the aim to improve adherence of exercises. The protocol is implemented in a mobile application, called SleepCare, that supports cognitive behaviour therapy for insomnia. The interaction design of the application is centred around the paradigm of Talk-and-Tools. Talk represents personalized conversations with a virtual e-coach, while Tools are represented by specialized modules that form a coherent structure of input and output facilities. We focus on the technique of sleep restriction, an intensive form of behaviour change that requires strong perseverance from the user. Both user and e-coach may negotiate about the attributes of the sleep restriction technique, here the duration of the total time in bed. The algorithm that calculates the negotiation space is personalized on the basis of previous sleeping behaviour of the coachee, the stage of the program and general constraints of the insomnia therapy. The system is evaluated by means of automated simulations and data from a randomized controlled trial. More than $50 \%$ of the subjects who did sleep restriction as a behaviour changing exercise used the protocol in the first round and negotiated with the system about an agreed time in bed. We expect that negotiation includes a number of important adherence enhancing strategies in e-coaching: increase of the coachee's autonomy, a sense of shared decision making and commitment.
\end{abstract}

Persuasive Technology, Conversation, Negotiation, E-Coaching, Cognitive Behaviour Therapy, Insomnia, Sleep Restriction.

\section{INTRODUCTION}

In parallel with contemporary technological and health care developments, a new field of interaction technology has emerged: so-called 'persuasive technology'. On the basis of motivation and behaviour models from psychology and social sciences, technological systems are introduced that support users in changing attitudes, thinking, and behaviour through technology (Fogg, 2003). Relatedly, a new terminology was adopted: not only are computer systems user-friendly, they must be cooperative, trustful, and able to build a relationship with users (Bickmore \& Picard, 2005; Reeves \& Nash, 1996). The role of the computer has changed from a command receiver to a supportive advice giver with respect to a user's behaviour change.

The goal of this paper is to present negotiation as a persuasive interaction strategy in automated mobile e-coaching systems for self-management of behaviour change (Lin \& Kraus, 2010). The central idea in self-management approaches to behaviour change is that individuals invest a reasonable amount of effort and time in prescribed activities. However, as in prescribed medical interventions, low adherence is an ever present and complex problem. People may enthusiastically start a particular self-help therapy and, subsequently, discover that the exercises require extensive self-discipline and stamina. As a result, they get into a downward motivation spiral and the therapy is terminated prematurely. This seriously undermines the outcome of behaviour change therapies (Vermeire et al., 2001). Preventing this process or adequately responding to it is a necessary element in any behaviour change intervention.

In this paper, we assume that computer systems contain only a fraction of the knowledge necessary to offer an adequate tailored intervention. Society and daily lives of people are highly contextual and characterized by a growing degree of uncertainty. What counts as a solution for one person may not matter as a solution for another. Consequently, ecoaching systems should be cautious in offering rigid solutions.

Negotiation enables patients to be involved in a shared decision making process of the attributes of their treatment (Kamphorst \& Kalis, 2015; Sandman, 2009). An important advantage of applying negotiation is the support of the patient's autonomy and, as a result, an improvement of the relationship between patient and professional by levelling of the patients' power and a decrease in informational asymmetry. Supporting the patient's autonomy may therefore increase the intrinsic motivation to follow the treatment plan (Zuckerman, 1978) and may 
contribute to positive patient outcomes, such as satisfaction and improvements in functional status (Charles et al., 1997; Legare, 2008).

In addition, by making a shared decision, patients explicitly commit themselves to the therapy plan which, in line with Cialdini's commitment and consistency principle, may lead to the tendency to act accordingly (Schulman-Green et al., 2014; Cialdini, 1993). It is therefore expected that the inclusion of shared decision making strategies in mobile ecoaching systems may have a positive effect on therapy adherence.

We implemented a negotiation protocol in a fully automated virtual e-coaching system called SleepCare which offers a tailored program on an Android smartphone in the domain of Cognitive Behaviour Therapy for Insomnia. The program was tested in a randomized controlled trial on 151 participants with insomnia complaints (Horsch et al., 2017). We observed that people that received the program improved a moderate to large degree on insomnia severity (Cohen's $d=0.66$ ) compared to people in a wait-list condition.

While we demonstrated the system's efficacy in the trial, we only briefly elaborated on how the negotiation strategy was implemented. Therefore, we will focus in this paper on the design of the negotiation protocol that was applied in the SleepCare system. In Section 2, we describe insomnia therapy and the basic elements of the SleepCare system. Section 3 provides the negotiation protocol and the implementation in the SleepCare system. Section 4 presents a brief evaluation of the protocol in SleepCare using simulation techniques and empirical data from a randomized controlled trial.

\section{INSOMNIA THERAPY AND THE SLEEPCARE SYSTEM}

\subsection{Cognitive behavioural therapy for insomnia}

The therapy implemented in the SleepCare system is based on cognitive behaviour therapy for insomnia (CBT-I) (Morin \& Espie, 2003; Edinger \& Carney, 2008). CBT-I is a collaborative and individualized program that helps individuals to identify unhelpful thoughts and behaviours, and to learn or relearn healthier skills and habits with respect to sleep. It is an effective form of treatment for insomnia in both classic face-to-face therapy (Van Straten et al., 2017) and in a computerized form (Zachariae et al., 2016).

CBT-I consists of several types of techniques that aim at improving sleep; examples are 'stimuluscontrol' (association of the bed with sleeping), sleep hygiene (control the environment and behaviours prior to sleeping, light, sound, alcohol and coffee use, etc.), relaxation, sleep education (in- formation transfer with respect to normal sleep and sleep problems) and sleep restriction (restricting the time in bed).

Computerized forms of CBT-I range from guided self-help (Van Straten et al., 2013) to fully automated internet-based programs (e.g., Espie et al., 2012). An overview of sleep technology is presented in Ko et al., (2015) and categorized by delivery platform, such as mobile device apps, wearable devices, embedded devices, accessory devices and conventional desktop/website resources. Mobile device apps, such as Sleep Cycle, SleepBot, Sleep As Android, usually focus on sleep tracking and waking and sleep inducing. Sleep tracking is directed at the observation of individual sleep characteristics, such as frequency, duration, or quality of sleep; this can be done by automatic sensing, manual input, or both. Waking and sleep-inducing technology supports people to wake up in the morning or to go to sleep at night. Currently, besides SleepCare, only one stand-alone app delivers a full CBT-I program ('CBT-I Coach'; see e.g., Koffel et al., 2017), but the app is developed as a supporting device while receiving clinician-delivered CBT-I. In general, a major problem is that a proliferation of sleep applications has arisen regardless of real user needs and that, in contrast to SleepCare, none of the algorithms applied in these mobile devices is validated by scientific studies or scientific literature (Ong \& Gillespie, 2016).

\subsection{The sleep restriction technique}

In this paper, we focus on the sleep restriction technique of CBT-I, which is considered as one of the most effective components (Miller et al., 2014). In sleep restriction the time in bed is restricted to the time a person is actually sleeping. For instance, if a person is in bed for about 9 hours a night but is sleeping only 6 , the time in bed will be restricted to 6 hours. At the same time a regular bed- and rising time is implemented. By maintaining a strict bedtime schedule, first a person's sleep debt is increased. This results in a higher sleep pressure that in turn improves the sleep efficiency, i.e. the proportion of sleep during the time spent in bed. When sleep efficiency increases, the time in bed can be lengthened in small steps until a desired sleep schedule is accomplished. Sleep restriction is intense, requires a considerable amount of the individual's perseverance and has several detrimental effects before sleep actually improves (Kyle et al., 2015). Therefore, the technique requires effective persuasive strategies to persuade the client to adhere to the exercises, in particular when a human coach is replaced by a mobile system.

\subsection{The SleepCare e-coaching system}

The SleepCare system represents an automated e-coach that applies a number of persuasive 
strategies, such as frequent feedback and adaptation of the therapy to the client's needs and abilities (Horsch et al., 2012; Beun, 2016). The system helps to set goals, develop a treatment plan and reminds the individual of scheduled assignments.

The interaction design of the e-coach is centred around the paradigm of Talk-and-Tools (Beun et al., 2017). This paradigm distinguishes between two types of interaction with our surrounding world: a symbolic and a physical one. The essential difference is that interactions of the first type need a (human) interpreter who can bridge the gap between the symbols and their actual meaning and purpose, while interactions of the second type are related in a more direct manner to (human) perception and action (e.g., Hutchins, 1989). This enables a human user to view interaction with computer systems as (i) a conversation with a virtual intermediary, (ii) a sequence of direct manipulations and observations of a virtual world or (iii) a combination of these interaction types. A combination of these types was already applied in the design of, for instance, SHRDLU (Winograd, 1971), the DenK-system (Ahn et al., 1995) and Collagen (Rich et al., 2001).

In SleepCare, the symbolic type is represented by personalized dialogues that mimic the conversational behaviour of the e-coach, such as natural language consultations, and the physical one by specialized tools that support particular tasks within the application domain, such as an electronic sleep diary or a relaxation module. The basic coaching process in SleepCare can, therefore, be considered as a series of conversations between two individuals - the e-coach and the coachee, and consists of three phases: opening, intervention and closure (see Figure 1; Beun et al., 2014). In the opening phase the e-coach and coachee align expectations about the therapy and construct a shared commitment with respect to the general treatment plan. In the intervention phase, the coachee is offered the actual therapy and conducts a combination of different CBT-I techniques. During the closure phase, the treatment is evaluated and finished, and, if necessary, the coachee can be referred to a professional.

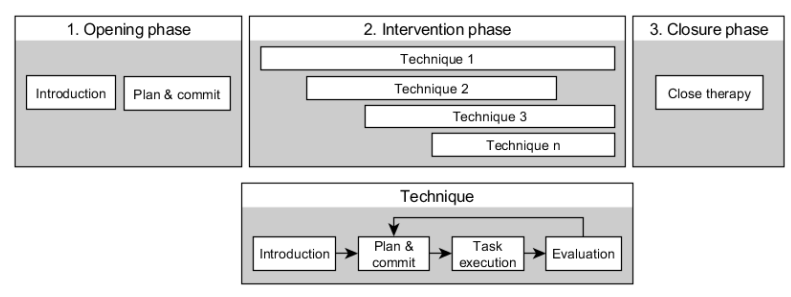

Figure 1: Basic coaching process [Beun et al 2014]

Each technique offered during the intervention phase consists of several stages: introduction, plan \& commit, task execution and evaluation (see Figure
1). In the first stage, the technique is explained to the coachee. Next, a plan is constructed with the coachee for the next period, usually a week, resulting in a shared commitment. While working with the assignments in the task execution stage, an evaluation of the progress is scheduled periodically to provide feedback on the coachee's adherence to the plan. After evaluation, the plan may be revised and committed to for the next period until goals are reached or the treatment ends. The introduction (and evaluation) of an assignment is provided to the coachee in the form of a conversation.

Conversations are presented in a WhatsApp kind of structure. To cover the main interaction needs in CBT-I, 18 different conversational types were included (e.g., introduction, planning and commitment, evaluation). These conversations can be considered as tree like structures where selections of the coachee or the value of particular sleep and behaviour data causes a branch. Conversations have some flexibility with respect to the information content, the surface structure of the expressions, and their timing. The flexibility of the content and surface structure of the turns is determined by branches in the tree and the variables in the templates. A particular branch in the tree may be selected on the basis of the coachee's choice in a number of pre-defined options or on the basis of contextual information, such as the coachee's sleep efficiency. Also, variables in the templates are assigned a particular value based on contextual information, such as time of day (e.g., 'Good morning', 'Good evening') or coachee information (e.g., 'Your sleep efficiency is 79\%'). Conversations are generated using XML-based predefined templates for describing the discourse moves of the e-coach and the multiple-choice response options for the coachee (Fitrianie et al., 2015).

Since interruptions and disrupting stimuli are common in mobile applications, conversational turns are as concise as possible and offered in small chunks. They can also be continued later by the coachee at a more convenient moment.

\section{NEGOTIATION IN SLEEPCARE}

In game theory, negotiation is often considered between two opposing participants that apply a negotiation strategy to maximize the individual outcome of the process (Osborne \& Rubinstein, 1994). In the context of a coaching program, however, we should be careful in applying game-theoretical strategies for negotiation. Here, the e-coach and coachee will be considered as cooperative partners with a common long-term goal (e.g., improving sleep quality of the coachee), but having incomplete information about the other and/or the domain of discourse, and possibly conflicting intermediate 
short-term goals (e.g., shorten the total time in bed).

\subsection{Terminology}

In SleepCare a negotiation round boils down to a sequence of related dialogue turns, the so-called proposals, where e-coach and coachee try to achieve mutual agreement about the attributes of a technique. In principle, negotiation may be applied to various attributes, such as duration, frequency and starting time; in SleepCare, we assume a single-variable negotiation process about the duration of the time spent in bed (TIB) of the sleep restriction technique, rounded to quarters of an hour. During a negotiation round, a limited number counter-proposals can be put forward by both conversational partners until eventually a particular proposal is mutually accepted or rejected by the coachee.

A negotiation strategy pertains to the content and the number of proposals. Depending on the coachee's stages of prior commitment and experience with the sleep restriction technique, we distinguish three types of strategies. In stage 1, there is no prior commitment by the coachee with respect to the technique; in stage 2 , there is commitment to the technique without prior experience of the behaviour change; in stage 3 , there is both commitment to the technique and prior experience of the behaviour change. In other words, in stage 3 new bedtimes have been experienced, evaluated and compared with the coachee's previous commitment, and possibly adapted as a result of a new negotiation round. Usually, stage 3 is achieved after three weeks.

Table 1. Relevant sleep parameters

\begin{tabular}{|c|c|c|}
\hline Name & $\begin{array}{l}\text { Abbre- } \\
\text { viation }\end{array}$ & Calculation \\
\hline Total Time Awake & TTA & Sleep diary \\
\hline Time In Bed & TIB & Rise Time - Bedtime \\
\hline Average Time in Bed & TIB $B_{\text {aver }}$ & $\sum_{n=1}^{7} T I B_{n} /_{7}$ \\
\hline Total Sleep Time & $T S T$ & TIB-TTA \\
\hline $\begin{array}{l}\text { Average Total Sleep } \\
\text { Time }\end{array}$ & $T_{S T} T_{\text {aver }}$ & $\sum_{n=1}^{7} T S T_{n} / 7$ \\
\hline Sleep Efficiency & $S E$ & $\left(T S T /{ }_{T I B}\right) * 100 \%$ \\
\hline $\begin{array}{l}\text { Average Sleep Effi- } \\
\text { ciency }\end{array}$ & $S E_{\text {aver }}$ & $\sum_{n=1}^{7} S E_{n / 7}$ \\
\hline
\end{tabular}

The content of the various proposals depends on the negotiation space which is defined as the set of acceptable TIB-values by the e-coach, ranging from the lowest acceptable $\mathrm{TIB}\left(\mathrm{TIB}_{\min }\right)$ to the highest acceptable TIB ( TIB $\left._{\max }\right)$. The negotiation space is determined by a) the actual sleep behaviour, b) the coachee's experience with and adherence to the sleep restriction technique and c) general constraints posed by the CBT-I guidelines. Information about the actual sleep behaviour is based on an electronic sleep diary that has to be filled in daily by the coachee (see also the Table 1 for relevant sleep parameters).

Table 2. Calculation of the negotiation space in the first round. Numbers are in hours.
(1) in all cases $5 \leq \mathrm{TIB} \leq 9$
(2) $\mathrm{TIB}_{\min }=\mathrm{TST}_{\text {aver }}$
(3) $\mathrm{TIB}_{\max }=$ average $\left(\mathrm{TIB}_{\text {aver }}, \mathrm{TST}_{\mathrm{aver}}\right)$
(4) if $\mathrm{TIB}_{\text {aver }} \geq 8$ then $\mathrm{TIB}_{\max } \leq \mathrm{TIB}_{\mathrm{aver}}-1$
(5) if $\left(\mathrm{TIB}_{\mathrm{aver}} \geq 8\right.$ and $\left.\mathrm{TST}_{\mathrm{aver}}<4\right)$ then $\mathrm{TIB}_{\max }=6$
(6) if $\left(\mathrm{TIB}_{\text {aver }} \geq 9\right.$ and $\left.\mathrm{TST}_{\text {aver }}<4\right)$ then $\mathrm{TIB}_{\max }=7$
(7) if $\left(\mathrm{TIB}_{\text {aver }}-\mathrm{TST}_{\mathrm{aver}}>4\right.$ and $\left.\mathrm{TST}_{\text {aver }} \geq 4\right)$ then
$\mathrm{TIB}_{\max } \leq \mathrm{TST}_{\mathrm{aver}}+2$

Table 3. Example calculations of the negotiation space $\left(T I B_{\min }, T I B_{\max }\right)$ in the first round

\begin{tabular}{|c|c|c|c|}
\hline TIB $_{\text {aver }}$ & TST $_{\text {aver }}$ & TIB $_{\min }$ & TIB $_{\max }$ \\
\hline 8 hours & 6 hours & 6 hours & 7 hours \\
\hline 9 hours & 7.5 hours & 7.5 hours & 8 hours \\
\hline 11 hours & 3.5 hours & 5 hours & 7 hours \\
\hline
\end{tabular}

Since adherence can only be determined after a particular period of performing the technique, it cannot be included as a parameter in the first negotiation round (which usually appears after the first seven days monitoring of the sleep diary by the ecoach). As a general constraint and safety rule, $T_{I B} B_{\text {min }}$ is always equal to or more than 5 hours and $\mathrm{TIB}_{\max }$ is always equal to or less than 9 hours. The algorithm that calculates the negotiation space in the first round is based on a modified version of the sleep restriction protocol (Morin \& Espie, 2003; Kyle et al., 2015) and the experience of a focus group of insomnia experts (Horsch et al., 2017) (see Table 2 and Table 3).

\subsection{Interaction structure}

Negotiation is applied during the Plan \& Commit stage, i.e., after the introduction of the technique and before the execution of the assignments, and may be repeated after an evaluation session (see Figure 1). Only if sleep efficiency is below $85 \%$ the sleep restriction protocol is started.

Before the actual negotiation process starts, a short introduction is presented that explains the 'what', the 'how' and the 'why' of the sleep restriction technique (see Figure 2). In each negotiation round, the e-coach starts with the first optimal proposal from the e-coach's view, which equals TIB $_{\min }$ (which is $\mathrm{TST}_{\text {aver }}$ with a minimum of 5 hours in all cases). The coachee can respond to the proposal in three 
ways: agree, make a counter-proposal, or stop with the negotiation process. If the coachee chooses to make a counter-proposal (see Figure 3), a limited set of options is presented as multiple choice buttons, including $\mathrm{TIB}_{\max }$; the e-coach accepts the proposal if it is within 30 minutes from the ecoach's first proposal. If the coachee chooses an option that is more than 30 minutes from the first proposal the negotiation continues. If the coachee chooses to stop, the e-coach makes a final offer to attempt persuading the coachee to continue.

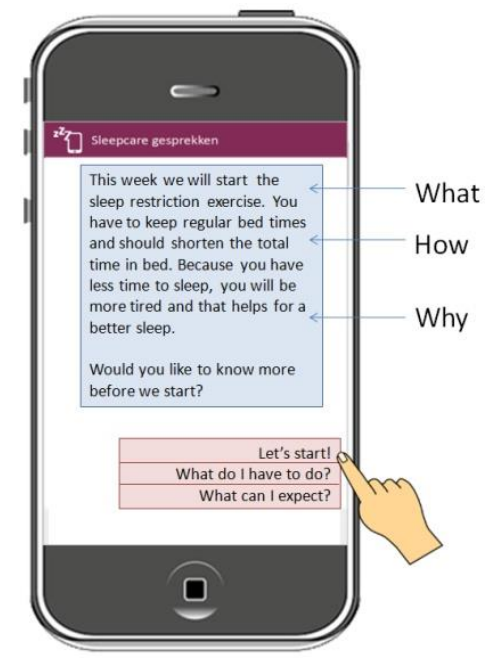

Figure 2: Introduction of the sleep restriction exercise (original conversation in Dutch).

In Figure 4, the final dialogue turns in a successful negotiation process are presented. Note that the options for TIB are now more fine grained, because the size of the negotiation space has decreased. After mutual agreement about the duration of TIB, the e-coach proposes concrete bed- and risingtimes. Within the limit of the agreed TIB, the coachee is free to change these bed-and rising times; this is not considered as a part of the negotiation process.

Each proposal from the e-coach is accompanied by persuasive messages, such as the information source of the proposal or the negative consequence of not accepting a previous proposal (Fogg, 2003; Cialdini, 1993). To avoid unnecessary lengthy dialogues and an increased risk for interruptions, the maximum number of proposals presented by the e-coach is two. When this limit is reached, the e-coach always agrees with the latest offer made by the coachee (as long as it is within the accepted limits).

If $\mathrm{TIB}_{\max }$ is not accepted in the first round, the ecoach will offer the possibility to reschedule the negotiation round and try to negotiate again the next day. If the negotiation fails twice, the technique will be cancelled. In that case, the sleep restriction technique will be rejected as part of the CBT-I program. Given the current limitations of conversations in mobile applications, we did not include a discussion about the reasons behind the coachee's wish to stop the sleep restriction technique.

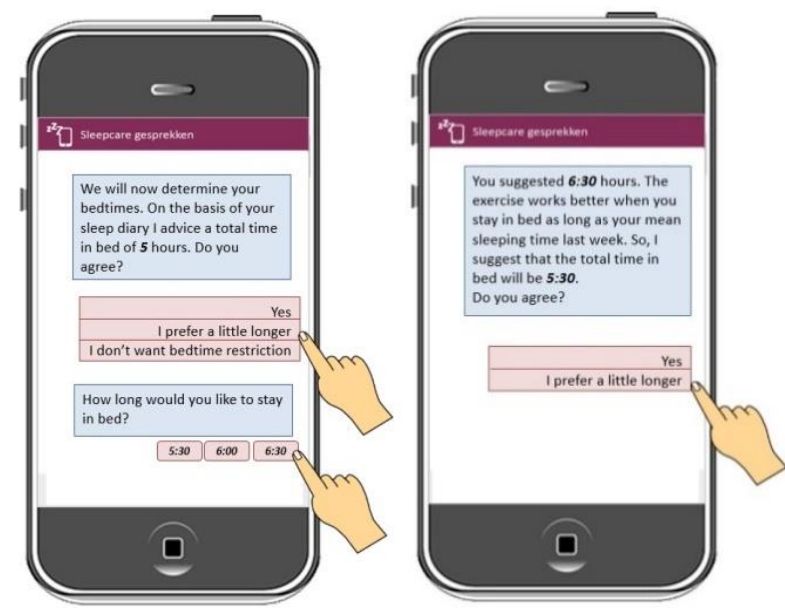

Figure 3: First proposal by the e-coach and a counterproposal by the coachee in a negotiation round (original conversation in Dutch). Note that the second pair of turns in the left picture appears after the coachee has provided a response to the first turn.

\subsection{Follow up rounds}

In the second round, i.e. right after stage 2, the advised TIB will in principle be the same duration as the week before. However, an important difference with stage 1 is that the coachee has now committed him/herself to an agreed behaviour change and hopefully practiced the technique in the first week. So, in stage 2, information about the coachee's adherence becomes available and a reward may be given on the basis of the coachee's performance.

In case the coachee's sleep efficiency is now more than $85 \%, 15$ minutes are added to $\mathrm{TIB}_{\min }$; in other words, the coachee is rewarded to stay in bed an extra 15 minutes. There are now three options for negotiation: $\mathrm{TIB}_{\min },+15$ and +30 minutes, in other words, the negotiation space is now 30 minutes. Based on the adherence information, the e-coach uses different scripts to explain the advice.

In the third round and further, i.e. after stage 3, there is even more information available with respect to the aimed behaviour change and the coachee's experience with the technique. The basis for the algorithm that calculates $\mathrm{TIB}_{\min }$ is (the change in) the average sleep efficiency in the last week and the week before last week together with the information about the coachee's adherence (see Table 4). In general, 15 minutes is subtracted from the previous TIB $_{\text {min }}$ if sleep efficiency is below $85 \%$ and 15 minutes is added if sleep efficiency is more than $85 \%$. If people do not adhere (in our case, $\operatorname{TIB}_{\text {aver }} \geq \mathrm{TIB}_{\text {agree }}+1$ hour), then an extra 15 minutes is added to $\mathrm{TIB}_{\mathrm{min}}$. When sleep efficiency 
decreases, $\mathrm{TIB}_{\min }$ will be the same as an earlier agreed proposal that resulted in an improved sleep efficiency. When sleep efficiency is improving, but between $80 \%$ and $85 \%$, the agreement from the previous round will be continued. At this stage, the negotiation space is small, because often improvement in sleep efficiency has started and the coachee should adhere to a strict schedule.

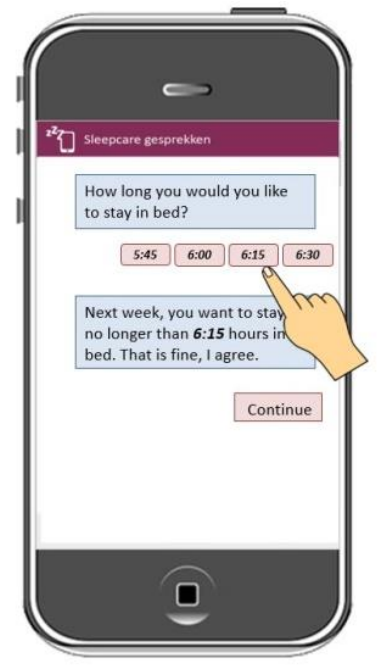

Figure 4: The final dialogue turns in the negotiation process (original conversation in Dutch).

The dialogues and the algorithm that calculates the negotiation space were developed in close collaboration with the insomnia expert who participated in the SleepCare-project (JL).

\section{EVALUATION OF THE NEGOTIATION PRO- TOCOL}

To evaluate the acceptability of the system, we now turn to the testing and user evaluation phases. We distinguish between two parts: (a) expert analysis by running simulations and preliminary user evaluations and (b) a randomized controlled trial (RCT) with insomnia patients. Below, we will summarize the methodology and some of the main findings.

\subsection{Simulation of scenarios and tool evaluation}

An automated testing environment was developed that consisted of the SleepCare app and a simulator (Griffioen-Both et al., 2016). The simulator imitated the coachee's interactive behaviour with the app, and allowed to playback pre-recorded and predefined actions.

In total, 32 scenarios were tested to verify the negotiation process; we varied the coachee's responses, the sleep behaviour measured by the electronic diary and the adherence to the program. To make sure that a large variety of realistic sleep data was tested and evaluated, the scenarios and the expected behaviour of the e-coach were de- signed together with the insomnia expert in our team (JL).

The recordings from the simulations were compared to the expected behaviour and shown to several other insomnia and communication experts. This resulted in minor adjustments of the e-coach's responses. For example, additional warnings were implemented when the coachee's sleep diary showed an average total sleep time of less than five hours, including a higher time in bed proposal by the e-coach. We also shortened the maximum number of negotiation proposals by the e-coach (from three to two), after receiving feedback about the length of the conversation.

In addition to these simulations, we evaluated various tools of the system with 10 people (e.g., filling in the sleep diary, performing relaxation exercises, rescheduling the exercises) separately from the actual program (see Beun et al., 2017). These evaluations resulted in 39 suggestions for improvement. In general subjects found the tools easy to learn, but it should be noted that the perceived usefulness of these tools reported by subjects that had a sleeping problem was considerably higher than those not having a sleeping problem.

In the final preparation for the RCT, a pilot experiment was conducted where 24 people started using the app in daily life. Here, the focus was on the risk of a decrease in the participants' sleep efficiency and sleep quality and the occurrence of technical failures. The results of the pilot experiment gave us enough confidence to start the actual RCT as a next step in the research process. At this stage, we did not evaluate the negotiation strategy separately from the total program.

\subsection{Results from the RCT}

Empirical results about the negotiation process were inferred from the RCT that was performed to test the efficacy of the total SleepCare program (Horsch et al., 2017). In the trial, the app-condition was compared with a wait-list group. Participants in the wait-list group received the app about seven weeks later after they completed a post-test questionnaire. In general, the results showed significant interaction effects favouring the app condition on insomnia severity and sleep efficiency. These improvements were also retained in a 3-month followup. After the post-test the wait-list group also received the app. Here, we discuss negotiation data from the app-group and the wait-list group combined (in total $n=118$ ).

Of the 118 participants, 46 never started a sleep restriction conversation and, consequently, did not apply the technique. Of these 46 participants 17 reported a sleep efficiency greater than $85 \%$ and were excluded (see also section 3.2); the remaining 29 participants did not perform the first sleep 
restriction conversation: 4 due to technical issues, 19 because they stopped using the app in the first week (i.e. before the first conversation about the start of the sleep restriction technique) and 6 continued using the app, in particular the sleep diary, but did not start any of the conversations.

The flow of the participants' negotiation process through 7 rounds is displayed in Figure 5.72 participants (i.e., 118-46) were offered the sleep restriction dialogue (SR_Intro in Figure 5); 4 refused the technique during the first sleep restriction conversation.

Of the remaining 68 participants who made a commitment for bedtimes in the coming week, 31 agreed with the first proposal of the e-coach and 37 negotiated about the time in bed. Of these 37 participants, 12 were talked down from their first counter-proposal. This means that in the end of the conversation, participants agreed to a shorter time in bed than they proposed themselves as their first proposal.

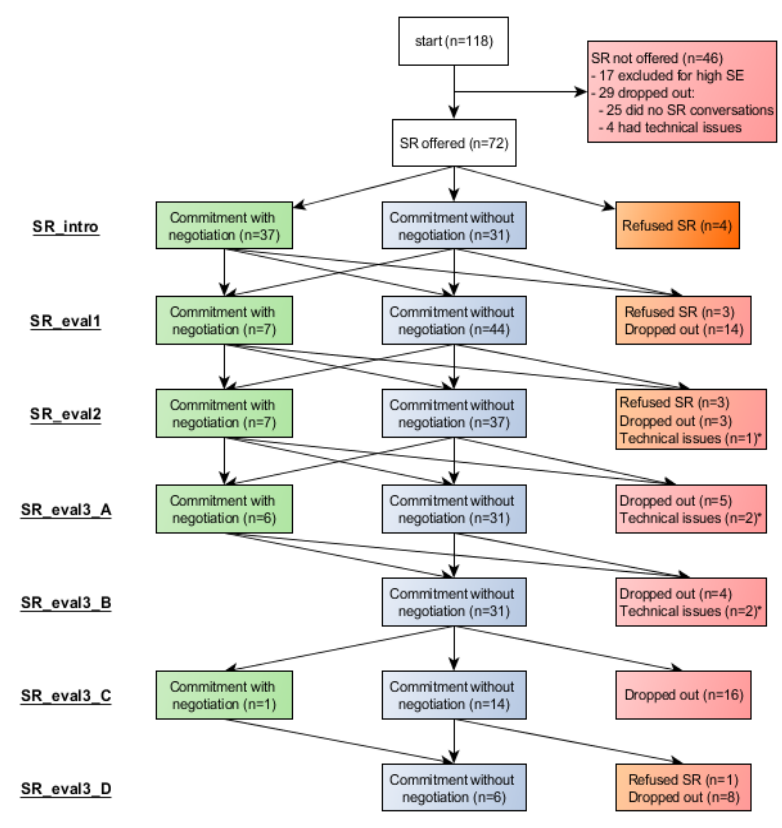

Figure 5: Flow diagram of participants through the different sleep restriction conversations (SR).

In Figure 5, we can see that most participants apply the negotiation in the first round and that only 6 subjects still apply the negotiation process in round 4 (SR_eval3_A). Also, most subjects dropped out from the sleep restriction technique in the end.

In Table 4, the measured average time in bed (TIBmes,aver), the average sleep efficiency ( $\left(\mathrm{SE}_{\text {aver }}\right)$, the average first proposal time in bed made by the ecoach ( $\left.T I B_{\text {min,aver }}\right)$ and the average time in bed agreement ( $\mathrm{TIB}_{\text {agr,aver }}$ ) are shown for non-negotiators and negotiators right after stage 1. A correlation was found between $\mathrm{TIB}_{\min }$ and the number of turns in the negotiations $(r=-.46 ; p<.001)$, indicating that participants are more likely to negotiate when $\mathrm{TIB}_{\min }$ is relatively low. Not surprisingly, $\mathrm{TIB}_{\text {agr,aver }}$ is higher in the negotiator group than for the nonnegotiators (non-negotiators always accept $\mathrm{TIB}_{\mathrm{min}}$ ). $\mathrm{TIB}_{\text {mes,aver }}$ and $\mathrm{SE}_{\text {aver }}$ did not differ between groups. We also did not find a correlation between the number of turns in SR_intro and adherence to the committed time in bed $(r=-.09 ; p=.46)$.

Table 4. Averages for the e-coach's first proposal and agreements right after stage 1.

\begin{tabular}{|l|l|l|l|l|}
\hline & $\begin{array}{l}\text { TIB- } \\
\text { mes,aver }\end{array}$ & $\mathrm{SE}_{\text {aver }}$ & $\mathrm{TIB}_{\text {min,aver }}$ & $\begin{array}{l}\text { TI- } \\
\mathrm{B}_{\text {agr,aver }}\end{array}$ \\
\hline $\begin{array}{l}\text { non- } \\
\text { negotiators } \\
\text { (n=31) }\end{array}$ & $8: 48$ & $82 \%$ & $7: 10$ & $7: 10$ \\
\hline $\begin{array}{l}\text { negotiators } \\
(n=37)\end{array}$ & $8: 42$ & $80 \%$ & $6: 40$ & $7: 26$ \\
\hline $\begin{array}{l}\text { average } \\
(n=68)\end{array}$ & $8: 45$ & $81 \%$ & $6: 54$ & $7: 19$ \\
\hline
\end{tabular}

When the coachee refused sleep restriction during the first sleep restriction conversation, the e-coach responded by making a final offer of 30 extra minutes. In total, 14 people were offered the extra 30 minutes, 10 people agreed with the latest offer and 4 people still refused (i.e. $71 \%$ was brought back into the program). In the evaluation conversations (SR_eval in Figure 5), the coach did not propose an additional 30 minutes, but tried to convince the coachee by explaining the importance of the sleep restriction technique. In all evaluation conversations, 20 participants were shown the explanation, 13 of them decided to continue with sleep restriction, 7 people refused.

During the RCT, we received over 900 emails about various aspects of the experiment. Participants asked questions, reported problems and provided suggestions for improvement, but none of these emails referred to the negotiation strategy. Opinions about the app diverged widely. For instance, someone reported that 'Bedtime restriction was a revelation', while another complained that 'Bedtime restriction didn't feel good, because the influence on daily life is huge' (translations from Dutch). Other people complained about the lack of a human presence ('I miss a real person to talk to'), while yet another was considerably more positive ('Maybe it sounds strange, but I had the feeling of building a bond with my coach, although I am aware that it is just an algorithm'). We were unable to link these email-statements to the actual use of the negotiation strategy.

\section{DISCUSSION}

Our starting point in the protocol was that e-coach and coachee should be considered as cooperative 
partners that aim at a common goal, with possibly intermediate conflicting goals. Both partners could negotiate about the attributes of the sleep restriction technique in insomnia therapy, in our case the duration of the time in bed (TIB). The algorithm that calculates the negotiation space was personalized, and based on previous sleeping behaviour of the coachee, the stage of the program and general constraints on the insomnia therapy.

In the negotiation protocol, we distinguished between the number and the content of the proposals. On the basis of results from the simulations and in order to avoid unnecessary lengthy dialogues and an increased risk for interruptions in the context of mobile interaction, we decided that the maximum number of proposals presented by the e-coach was two. When this limit was reached, the e-coach always agreed with the latest offer made by the coachee.

Concerning the content, the e-coach always started with $\mathrm{TIB}_{\min }$, i.e. the proposal that is theoretically most effective to solve the sleeping problem. The downside of $\mathrm{TIB}_{\min }$ is that it usually requires strong perseverance from the coachee and, therefore, may be less effective from a persuasive point of view. We expected that including negotiation and offering an acceptable TIB-window (i.e. the negotiation space) would include a number of important adherence enhancing strategies: increase of the coachee's autonomy, a sense of shared decision making and commitment (Schulman-Green et al., 2014; Cialdini, 1993). Clearly, when the behaviour change requires strong willpower, there will always be a trade-off between the most effective value of a particular attribute of the behaviour change and the coachee's ability or motivation to adhere to that change.

Results from an RCT showed that the SleepCare system was effective in general (Horsch et al, 2017) and that, if people were offered sleep restriction, more than half of the subjects used the possibility to negotiate (37 out of 68 subjects); 4 people refused the technique from the first sleep restriction conversation and 31 accepted the first offer (68-37). After the first round, however, the number of subjects that still negotiated dramatically decreased, possibly because coach and coachee were successfully aligned. In other words, there seemed to be a strong need by some users to discuss and adapt the attributes of the behaviour change at the start of the program. The result that negotiation is used in particular when $\mathrm{TIB}_{\min }$ is relatively low underlines our expectation that adaptation is desired when the behaviour change requires more perseverance.

We did not find a correlation between the negotiation behaviour and the adherence to the committed time in bed. There may be several reasons for not identifying this relationship. One possibility is that there are two separate groups. One group that benefits from negotiation because otherwise they dropout, and yet another group that believes that the first offer is reasonable and, therefore, adheres to the intervention without a need to negotiate the exercise attributes. In the current study, we did not have the statistical power to investigate these groups separately.

It should be stressed, though, that the goal of the RCT was to test the efficacy of the complete SleepCare system, not specifically focusing on the negotiation module. Because negotiation was one of the many persuasive strategies applied in the system (see Beun et al., 2016), only an app group and a waiting list group were compared. An advantage is that we have data from an experiment that, to some extent, matches a real-world situation. On the other hand, we only have data on the use of the negotiation protocol and results are lacking from a full-fledged experiment, more specifically a set up to test the relationship between adherence and negotiation. In such an experiment, two conditions could be compared, i.e., with or without negotiation, with adherence and therapy outcome as dependent variables.

\section{CONCLUSION}

In this paper, an automated negotiation process was presented as a persuasive interaction strategy for behaviour change applications in mobile devices. The negotiation protocol was implemented in a mobile application, called SleepCare, that supports cognitive behaviour therapy for insomnia. We focused on the technique of sleep restriction, an intensive form of behaviour change that requires strong perseverance from the user over a period of about six weeks.

We started from the idea that a user interface can be modelled as a combined interaction structure of natural conversations and specified tools that support a specific task - the so-called Talk-and-Tools paradigm (Beun et al., 2017). Conversations mimicked the interactive behaviour of a virtual e-coach that discusses various aspects with the user about the therapy, while tools form a coherent structure of input and output facilities that enable the user to get access to relevant data (e.g., electronic sleep diary, exercise schedule). Qualitative data from email messages supported the idea of including a virtual e-coach, although in some cases users indicate the need for a human partner. We, therefore, believe that a conversational component, whether human or virtual, fills an important gap in the interaction design of persuasive systems.

We showed that the negotiation protocol can be built in a WhatsApp kind of conversational structure with multiple choice response buttons that represent the admitted proposals by the coachee. 
Results from an RCT showed that there is a need for interaction about and adaptation of the attributes of the behaviour change, in particular in those cases where the behaviour change requires strong perseverance.

Unfortunately, the available data from the RCT did not enable us to draw statistically valid conclusions with respect to an improvement of the users' adherence to the behaviour change as a result of the inclusion of a negotiation strategy. So far, we can only speculate that the availability of negotiation increased adherence and motivational factors such as perceived autonomy and shared commitment.

We believe that our negotiation approach is generalizable towards other e-coaching domains and that it will support coachees by maintaining their autonomy and increasing their adherence. E-coaching refers to a more explorative interaction style, a highly personalized and contextualized process of deliberation characterized by a continuous cycle of observation, feedback and adaptation. Since computer systems contain only a fraction of the knowledge necessary to offer an adequate tailored therapy, negotiation seems to be a promising interaction strategy for digital coaching programs that support automated self-help therapies and behaviour change.

\section{ACKNOWLEDGEMENTS}

This research is supported by Philips and Technology Foundation STW, Nationaal Initiatief Hersenen en Cognitie NIHC under the Partnership program Healthy Lifestyle Solutions. We would like to thank the anonymous reviewers for their valuable contribution.

\section{REFERENCES}

Ahn, RMC, Beun, RJ, Borghuis, T, Bunt, HC \& Overveld, CWAM van (1995) The DenKarchitecture: a fundamental approach to user interfaces. Al Rev 8, 431-445.

Beun, RJ, Griffioen-Both, F, Ahn, RMC, Fitrianie, S \& Lancee J. (2014) Modelling interaction in automated e-coaching: A case from insomnia therapy. In: Proc. of COGNITIVE'14. May 25-29, 2014, Venice, Italy.

Beun, RJ, Brinkman, WP, Fitrianie, S, GriffioenBoth, F, Horsch, C, Lancee, J \& Spruit, AGL (2016) Improving Adherence in Automated eCoaching: A Case from Insomnia Therapy. In: Proc. of PERSUASIVE 2016. Meschtscherjakov, A, De Ruyter, B, Fuchsberger, V, Murer, M \& Tscheligi, M (eds) LNCS vol. 9638, Berlin: Springer, 276-287.
Beun, RJ, Fitrianie, S, Griffioen-Both, F, Spruit, AGL, Horsch, C, Lancee, J \& Brinkman, WP (2017) Talk and Tools - The best of both worlds in mobile user interfaces for E-coaching. Personal and Ubiquitous Computing, 21(4), 661-674.

Bickmore, TW \& Picard, RW (2005) Establishing and maintaining long-term human-computer relationships. ACM Transactions on ComputerHuman Interaction, 12(2), 293-327.

Charles, C, Gafni, A \& Whelan, T (1997) Shared Decision-Making in the Medical Encounter: What Does It Mean? (Or It Takes At Least Two To Tango). Soc Sci Med, 44(5), 681-692.

Cialdini, RB (1993) Influence: Science and Practice. New York: Pearson.

Edinger, JD, \& Carney, CE (2008) Overcoming insomnia: a cognitive-behavioral therapy approach-therapist guide. New York: Oxford University Press.

Espie, CA, Kyle, SD, Williams, C, Ong, JC, Douglas, NJ, Hames, P, Oxon, MA \& Brown, JSL (2012) A Randomized, Placebo-Controlled Trial of Online Cognitive Behavioral Therapy for Chronic Insomnia Disorder Delivered via an Automated Media-Rich Web Application. SLEEP, 35(6), 769-781.

Fitrianie, S, Griffioen-Both, F, Spruit, S, Lancee, J \& Beun, RJ (2015) Automated dialogue generation for behaviour intervention on mobile devices. Procedia Comput Sci, 63, 236-243.

Fogg, BJ (2003) Persuasive Technology: Using Computers to Change What We Think and Do. Amsterdam: Morgan Kaufmann Publishers.

Griffioen-Both, F, Spruit, S, Fitrianie, S, Lancee, J \& Beun, RJ (2016) Testing for mobile e-health interventions. SAC'16, April 4-8, 2016, Pisa.

Horsch, CHG, Brinkman, WP, Eijk, RM van \& Neerincx, MA (2012) Towards the usage of persuasive strategies in a virtual sleep coach. Proc. of UKHCl 2012 Workshop on People, Computers \& Psychotherapy. Birmingham, September 10, 2012.

Horsch, CHG, Lancee, J, Griffioen-Both, F, Spruit, S, Fitrianie, S, Neerincx, Beun, RJ \& Brinkman, WP (2017) Mobile Phone-Delivered Cognitive Behavioral Therapy for Insomnia: A Randomized Waitlist Controlled Trial. J Med Internet Res, 19(4): e70.

Hutchins, EL (1989) Metaphors for interface design. In: Taylor, MM, Néel, F, Bouwhuis, DG (eds.) The structure of multimodal dialogue, North-Holland, 11-28.

Kamphorst, BA \& Kalis, A (2015) Why option generation matters for the design of autonomous ecoaching systems. Al \& Society, 30(1), 77-88. 
Ko, PR, Kientz, JA, Choe, EK, Kay, M, Landis, CA \& Watson, NF (2015) Consumer sleep technologies: a review of the landscape. J Clin Sleep Med,11(12), 1455-1461.

Koffel, E, Kuhn, E, Petsoulis, N, Erbes, CR, Anders, S, Hoffman, JE, Ruzek, JI \& Polusny, MA (2018) A randomized controlled pilot study of CBT-I Coach: feasibility, acceptability, and potential impact of a mobile phone application for patients in cognitive behavioral therapy for insomnia. Health Inf J, 24(1), 3-13.

Kyle, SD, Aquino, MRJ, Miller, CB, Henry, AL, Crawford, MR, Espie, CA \& Spielman, AJ (2005) Towards standardisation and improved understanding of sleep restriction therapy for insomnia disorder: A systematic examination of CBT-I trial content. Sleep Med Rev, 23. 83-88.

Legare, F, Ratte, S, Gravel, K \& Graham, ID (2008) Barriers and facilitators to implementing shared decision making in clinical practice: Update of a systematic review of health professionals' perceptions. Patient Educ. Couns., 73, 526-35.

Lin, R, \& Kraus, S (2010) Can automated agents proficiently negotiate with humans? Communications of the ACM, 53(1), 78-88.

Mitchell, MD, Gehrman, P, Perlis M \& Umscheid, CA (2012) Comparative effectiveness of cognitive behavioral therapy for insomnia: a systematic review. BMC Fam Pract, 13(40).

Miller, CB, Espie, CA, Epstein, DR, Friedman, L, Morin, CM \& Pigeon, WR (2014) The evidence base of sleep restriction therapy for treating insomnia disorder. Sleep Med Rev, 18(5), 415424.

Morin, CM, Bootzin, RR, Buysse, DJ, Edinger, JD, Espie, CA \& Lichstein, KL (2006) Psychological and behavioral treatment of insomnia: Update of the recent evidence (1998-2004), SLEEP, 29 (11), 1398-1414.

Morin, CM \& Espie, CA (2003) Insomnia. A clinical Guide to Assessment and Treatment, New York: Springer.

Ong, AA \& Gillespie, MB (2016) Overview of smartphone applications for sleep analysis. World $\mathrm{J}$ of Otorhinolaryngology-Head and Neck Surgery, 2(1), 45-49.

Osborne, MJ \& Rubinstein, A (1994) A Course in Game Theory. Cambridge (Mass): The MIT Press.

Reeves, B \& Nass, C (1996) The media equation: how people treat computers, television and new media like real people and places. Cambridge: CUP.
Rich, C, Sidner, CL \& Lesh, N (2001) Collagen: applying collaborative discourse theory to human-computer interaction. AI Mag, 22(4),15-25.

Sandman, $L$ (2009) The concept of negotiating in shared decision making. Health Care Analysis, 17(3), 236-243.

Schulman-Green, DJ, Naik, AD, Bradley, EH, McCorkle, R \& Bogardus, ST (2006) Goal setting as a shared decision-making strategy among clinicians and their older patients. Patient Educ Couns, 63,145-51.

Straten, A van, Emmelkamp, J, Wit, J de, Lancee, J, Andersson, G, Someren, EJW van \& Cuijpers, P (2013) Guided Internet delivered cognitive behavioural treatment for insomnia: a randomized trial. Psychological Medicine, 44(7), 1521-1532.

Straten, A van, Zweerde, T van der, Kleiboer, A, Cuijpers, P, Morin, CM \& Lancee, J (2017) Cognitive and behavioral therapies in the treatment of insomnia: A meta-analysis. Sleep Med Rev, 38, 3-16.

Vermeire, E, Hearnshaw, H, Royen, $\mathrm{P}$ van \& Denekens, J (2001) Patient adherence to treatment: three decades of research: a comprehensive review. J of Clin Pharm and Therap, 26, 331-342.

Winograd, T (1971) Procedures as a representation for data in a computer program for understanding natural language, MAC-TR-84, MIT Project MAC.

Zachariae, R, Lyby, MS, Ritterband, LM \& O'Toole, MS (2016) Efficacy of internet-delivered cognitive-behavioral therapy for insomnia: a systematic review and meta-analysis of randomized controlled trials. Sleep Med Rev, 30, 1-10.

Zuckerman, M, Porac, J, Lathin, D, Smith, R \& Deci, EL (1978) On the importance of selfdetermination for intrinsically motivated behaviour. Pers \& Soc Psych Bull, 4, 443-446. 